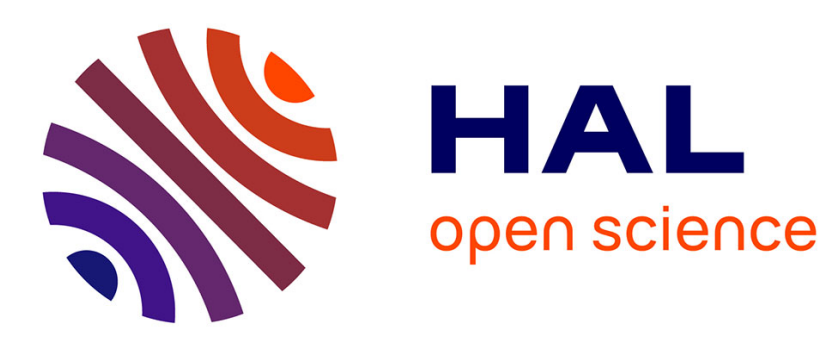

\title{
Coverage Extension Based on Incentive Scheduler for Mobile Relaying Nodes in Wireless Networks
}

Cédric Gueguen, Abderrezak Rachedi

\section{To cite this version:}

Cédric Gueguen, Abderrezak Rachedi. Coverage Extension Based on Incentive Scheduler for Mobile Relaying Nodes in Wireless Networks. Local Computer Networks (LCN), 2011 IEEE 36th Conference on, Oct 2011, Bonn, Germany. pp.303 - 306, 10.1109/LCN.2011.6115309 . hal-00676756v3

\section{HAL Id: hal-00676756 \\ https://hal.science/hal-00676756v3}

Submitted on 9 May 2012

HAL is a multi-disciplinary open access archive for the deposit and dissemination of scientific research documents, whether they are published or not. The documents may come from teaching and research institutions in France or abroad, or from public or private research centers.
L'archive ouverte pluridisciplinaire HAL, est destinée au dépôt et à la diffusion de documents scientifiques de niveau recherche, publiés ou non, émanant des établissements d'enseignement et de recherche français ou étrangers, des laboratoires publics ou privés. 


\title{
Coverage Extension Based on Incentive Scheduler for Mobile Relaying Nodes in Wireless Networks
}

\author{
Cédric Gueguen and Abderrezak Rachedi \\ University Paris-Est Marne-la-Vallée (UPEMLV) \\ Gaspard Monge Computer Science Laboratory (UMR 8049 LIGM) \\ Champs sur Marne, France \\ Email: \{gueguen, rachedi\} @univ-mlv.fr
}

\begin{abstract}
In this paper, we propose a new scheduler able to extend the wireless coverage by using an incentive approach for potential mobile relaying nodes. Indeed, the cost of cooperation can be expensive in terms of QoS and energy consumption which do not motivate the nodes to cooperate. Our incentive approach rewards the cooperative nodes. The percentage of cooperation is considered in the QoS management in order to incite the border nodes to cooperate and then to extend the wireless area. Moreover, the monitoring mechanism is proposed to correctly evaluate the cooperation rate of each node. The results show that not only the proposed solution allows the border nodes to cooperate without the negative impact but also enhance the QoS parameters.
\end{abstract}

Index Terms - Incentative Scheduling, Coverage extension, Cooperation, Quality of Service, opportunistic scheduling.

\section{INTRODUCTION}

The basic idea of the coverage extension area in wireless networks is to increase the network connectivity without increasing the infrastructure. This is one of the main applications of the cooperative communications in wireless networks. The coverage extension issue requires the cooperation of border mobile nodes to relay the packets of neighbouring nodes which are located outside of the base-station area. Many researchers dealt with the strategies to find the optimal placement for the relayed nodes in order to guarantee the Quality of Services (QoS) [1]. Other works dealt with the optimal number of hops of relayed nodes in wireless networks [2][3]. However, they assume that the relayed nodes by definition are fixed and cooperative which is not interesting in the case of a dynamic wireless network where the nodes freely move and potentialy selfish. No incentive approach is considered in these works. The relayed nodes must share their throughput with other neighbouring nodes which can impact their own packets transmission. In addition, the energy consumption of the relayed nodes is more important than the one of other classical nodes. They do not only transmit their own packets but also the packets of other neighbouring nodes. Therefore, the user of the potential relayed node can disable the cooperative functionality in order to keep the performance in terms of QoS only for its own transmission.

In this paper, we consider that the mobile relayed nodes are not part of the fixed wireless infrastructure. That is why the incentive strategy for potential mobile relay nodes is very important to consider in the design of the cooperation protocol. Our proposal is based on an incentive approach with QoS consideration for the mobile relayed nodes in order to extend the coverage area. This approach consists in increasing the priorities of the relayed nodes according to their cooperation rate. The idea is to reward the relayed nodes for their cooperation instead of penalized them by the cost of the cooperation. Consequently, the nodes do not have the benefits to select and act as selfish behaviour using their throughput only to transmit their own packets. Our proposed solution called "Coverage Extension based on Incentive scheduling" (CEI) is also build in a cross layer approach. Moreover incentive nodes to cooperate, the physical layer informations are used in order to take advantage of the time, frequency and multiuser diversity and maximize the system capacity close to the Shannon limit. Unlike the existing models, our solution can be widely implemented, evaluated and compared to the existing ressource allocation strategies like classical RoundRobin (RR) [4] and acknowledged MaxSNR [5][6].

This paper is organized as follows: in Section 2, we present the related works. Section 3 describes the proposed coverage extension protocol based on the incentive approach. The fourth Section presents the obtained simulation results and their analysis. Finally, Section 5 concludes the paper and presents our future works.

\section{RELATED WORK}

The conventional access methods like Round Robin (RR) [4] and Random Access (RA) are not adapted to the wireless environment and provide poor throughput. More recently intensive research efforts have been made in order to propose more efficient schedulers: the opportunistic schedulers. They preferably allocate the resources to the active mobile(s) with the most favourable channel conditions at a given time. Taking benefit of multiuser and frequency diversity in order to maximize the system throughput, all these schemes strongly rely on diversity for offering their good performances. One major scheduling algorithm has emerged and appeared in literature as the reference: Maximum Signal-to-Noise Ratio (MaxSNR).

In MaxSNR, priority is given at every scheduling event to the mobiles which have the greatest signal-to-noise ratio (SNR). It allocates the resource at a given time to the active mobile with the greatest SNR [5][6]. Taking profit of multiuser and frequency diversity, MaxSNR scheduling continuously allocates the radio resource to the mobile with the best spectral efficiency. Consequently, MaxSNR strongly increases 
the system throughput. Dynamically adapting the modulation and coding allows one to always make the most efficient use of the radio resource and come closer to the Shannon limit.

However MaxSNR does not take into consideration other aspect than throughput and particularly MaxSNR scheduling does not manage priority in order to favour cooperative mobiles. Consequently, no reward is guaranteed to cooperative mobiles. Their supplementary energy consumption and the personal throughput loss are not compensated. These results in a severe penalty for them which do not encouraged cooperative networks and coverage extension.

\section{The InCENTIVE SCHEDUleR AlgoRIthM}

The scheduler grants radio resources to each mobile as a function of its: channel state, currently cooperation ratio, network confidence percentage, traffic backlog. The knowledge of the channel state is supposed to be available at the receiver [7]. The channel attenuations are estimated by the access node based on the SNR. The CEI scheduling algorithm relies on weights that set the dynamic priorities for allocating the resource. These weights are built in order to satisfy two major objectives: to maximise system throughput and to encourage nodes to cooperate.

\section{A. System Throughput Maximization Parameter}

The CEI scheduler maximizes the system throughput in a MAC/PHY opportunistic approach. Data integrity requirements of the mobiles are enforced adapting the modulation scheme and the transmission power to the mobile specific channel state. At each scheduling epoch, the scheduler computes the maximum number of bits $m_{k, n}$ that can be transmitted in a time slot of subcarrier $n$ if assigned to a mobile $k$, for all $k$ and all $n$. This number of bits is limited by two main factors: the data integrity requirement and the supported modulation orders.

The bit error probability is upper bounded by the symbol error probability and the time slot duration is assumed to be equal to the duration $T_{s}$ of an OFDM symbol [5]. The required received power $P_{r}(q, k)$ for transmitting $q$ bits in a Resource Unit (RU) while keeping below the data integrity requirement $B E R_{\text {target }, k}$ of the service flow of mobile $k$ is a function of the modulation type, its order and the single-sided power spectral density of noise $N_{0}$. For QAM and a modulation order $M$ on a flat fading channel [8]:

$$
P_{r}(q, k)=\frac{2 N_{0}}{3 T_{s}}\left[\operatorname{erfc} c^{-1}\left(\frac{B E R_{\text {target }, k}}{2}\right)\right]^{2}(M-1),
$$

where $M=2^{q}$ and $\operatorname{erfc}$ is the complementary error function. $P_{r}(q, k)$ may also be determined in practice based on BER history and updated according to information collected on experienced BER.

The transmission power $P_{k, n}$ of mobile $k$ on subcarrier $n$ is upper bounded to a value $P_{\max }$ which complies with the transmission Power Spectral Density regulation:

$$
P_{k, n} \leq P_{\max }
$$

Given the channel gain $a_{k, n}$ experienced by mobile $k$ on subcarrier $n$ (including path loss and Rayleigh fading):

$$
P_{r}(q, k) \leq a_{k, n} P_{\max }
$$

Hence, the maximum number of bits $q_{k, n}$ of mobile $k$ which can be transmitted on a time slot of subcarrier $n$ while keeping below its BER target is:

$$
q_{k, n} \leq\left\lfloor\log _{2}\left(1+\frac{3 P_{\max } \times T_{s} \times a_{k, n}}{2 N_{0}\left[e r f c^{-1}\left(\frac{B E R_{\text {target }, k}}{2}\right)\right]^{2}}\right)\right\rfloor .
$$

We further assume that the supported QAM modulation orders are limited so that $q$ belongs to the set $S=$ $\left\{0,2,4, \ldots, q_{\max }\right\}$. Hence, the maximum number of bits $m_{k, n}$ that will be transmitted on a time slot of subcarrier $n$ if this $\mathrm{RU}$ is allocated to the mobile $k$ is:

$$
m_{k, n}=\max \left\{q \in S, q \leq q_{k, n}\right\} .
$$

MaxSNR based schemes allocate the resources to the mobiles which have the greatest $m_{k, n}$ values. This bandwidth allocation strategy maximizes the bandwidth usage efficiency but do not encourage nodes cooperation. In order to extend coverage area while preserving the system throughput maximization, a new parameter is added on $m_{k, n}$ which modulates these pure opportunistic resource allocation.

\section{B. Incentative Parameter}

The second major objective of the CEI is to incite nodes to participate to frame relay in order to extend the network coverage zone. This is achieved by extending the above crosslayer design to other layers. A new "Incentive Parameter" $\left(I P_{k}\right)$ is introduced based on the current estimation of the cooperation ratio:

$$
I P_{k}=\frac{R_{k}}{D_{k}}=\frac{D_{k}+\sum_{i=0 \ldots i=K}^{i} D_{k i}}{D_{k}},
$$

where $R_{k}$ is the global amount of data transmitted by the mobile $k$. It is the sum between $D_{k}$, the amount of data transmitted to the mobile $k$ for its own requirement and $D_{k i}$, the amount of data transmit to the mobile $k$ for a mobile $i$ (then these data will be relayed to mobile $i$ by mobile $k$ in the relaying subframe). This information could be directly monitored by the access point, or signalled by each mobile to access point.

We also define the cooperation ratio $C_{k}$ as the number of packets that the mobile $k$ is ready to relay for other mobiles when it receives 100 packets for its own consumption, for exemple:

- if mobile $k$ relay no traffic out of the cell, $C_{k}$ equal $0 \%$;

- if it is ready to relay 50 packet out of the cell since it receive 100 packets for its own consumption, $C_{k}$ equal $50 \%$

- if the mobile relay as much packet out of the cell that its own received for its own consumption, $C_{k}$ equal $100 \%$. 
Supposing that there are always packets to relay out of the cell, the $I P_{k}$ will be respectively for these three cases equal to $1,1.5$ and 2. Consequently, based on the ressource allocation on $I P_{k}$ allows to give more priority on mobiles which cooperate to extend coverage zone with frame relaying.

\section{Confidence Parameter}

We assume that each mobile signals its $R_{k}$ and $D_{k}$ to the access point. Thanks to this information, the CEI scheduler will make the adequate resource allocation rewarding the mobile for its cooperation degree. However in order to block malicious mobiles which could lie on this information, we introduced the last paramater called confidence parameter. The confidence parameter $T_{k}$ depends on the correspondence between the announced cooperative ratio and the observed forwarding ratio. This control is done by a monitor node (in our case the AP or cluser-head). Each $T_{k}$ is varies between 0 and 1 included. When the access point watching on $R_{k}$ and $D_{k}$ correspond to the annonced cooperative ratio, $T_{k}$ is set to 1 . On the contrary, when the mobile does not relay the announced amount of data for which it had previously received more priority, its $T_{k}$ is set to 0 for one round of scheduling in order to punish it. This assures a deterrent threat for mobile which would try to mislead the system.

\section{Global CEI Algorithm Description}

In the allocation process of a given time slot, the priority of a mobile with respect to another is determined by the magnitude of its CEI parameter :

$$
C E I_{k, n}=m_{k, n} \times \frac{R_{k}}{D_{k}} \times T_{k} .
$$

Based on the $m_{k, n}$ and $I P_{k}$ factor, the $C E I_{k, n}$ directly takes into account the channel states and the mobile behavior. Physical layer information is used with $m_{k, n}$ in order to take advantage of the time, frequency and multiuser diversity and maximize the system capacity. Cooperation information is exploited in a weighted system with $I P_{k}$ parameter that introduces dynamic priorities between mobiles for ensuring good awards to mobiles which help to extend the coverage zone. This results in an efficient scheme which guarantees a better network connectivity while avoiding tradeoff with the system capacity.

The $T_{k}$ paramater is an additionnal factor which allows to temperate $C E I_{k, n}$ value function of network confidence. Include $T_{k}$ parameter allows to be resistant to malicious nodes which would lie on its $\sum_{i=0 \ldots i=K}^{i} D_{k i}$. Thanks to this control parameter, no malicious mobiles will retransmit the packets which they have previously received supplementary priorities will be penalised and the good behavior are stimulate.

\section{PERformance Analysis}

In this section we evaluate by simulation the performance of the proposed CEI scheduling and we compare it with the classical Round Robin allocation and the well acknowledged MaxSNR scheduler. We consider four kinds of nodes: the first kind of nodes are selfish and do not relay packets $\left(C_{k}=0 \%\right)$, the second kind relay a few packets with $C_{k}=10 \%$, the third kind is more cooperative with $C_{k}=50 \%$ and the last kind of nodes is really network friendly with a high cooperative ratio of $100 \%$.

\section{A. Simulation setup}

We assume that each frame is formed by 128 subcarriers and 5 time slots. The channel gain model on each subcarrier considers free space Path Loss $a_{k}$ and multipath Rayleigh fading $\alpha_{k, n}^{2}$ [9]:

$$
a_{k, n}=a_{k} \times \alpha_{k, n}^{2} .
$$

where $a_{k}$ is dependent on the distance between the access point and mobile $k$ and $\alpha_{k, n}^{2}$ represents the flat fading experienced by mobile $k$ on subcarrier $n . \alpha_{k, n}$ is Rayleigh distributed with an expectancy equal to unity. Additionally, the maximum transmission power satisfies:

$$
10 \log _{10}\left(\frac{P_{\max } T_{s}}{N_{0}} \times a_{\text {ref }}\right)=31 \mathrm{~dB}
$$

and the BER target is equal to $10^{-3}$. With this setting, the value of $m_{k, n}$ is 3 bits when $\alpha_{k, n}^{2}$ equals one.

In order to be close to the reality, we consider that all mobiles run the same videoconference application. This demanding type of application generates a high volume of data with a high sporadicity and requires tight delay constraints which substantially complicate the task of the scheduler. The traffic load variation is done through increasing the mobile bit rate requirement of each mobile all together.

\section{B. Delay impact}

First we focus on the mean mobile packet delay provided by each scheduler function of different traffic loads keeping a special attention on their ability to encourage mobile cooperation with a low guaranteed delay. The obtained results are plotted in figure Fig. 1 with the mean throughput required by each mobile of the cell represented on the abscissa.

Figure 1(a) shows the case of RR with different cooperation ratios of nodes. We remark that the classical RR fails to promote cooperation activities. The RR fairly allocates the RUs to the mobiles without taking into account the effort of the cooperative mobile nodes which share their allocated resources with other nodes located out of the primary access point cell. Consequently, the more cooperative the nodes are, the less resources for their own transmission they have. Moreover, the RR does not take benefit of multiuser diversity which results in a bad utilization of the bandwidth and in turn, poor system throughput. Consequently, unacceptable packet delay is experienced even with relatively low traffic loads.

Figure 1(b) illustrates the obtained results in the case of MaxSNR with different cooperation ratios of nodes. We point out that even if a higher traffic load is supported with an acceptable packet delay, the cooperative nodes are not rewarded and their performance in terms of QoS are less compared to the non-cooperative nodes. 


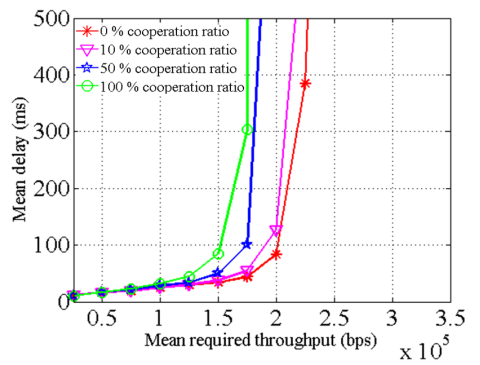

(a) With RR.

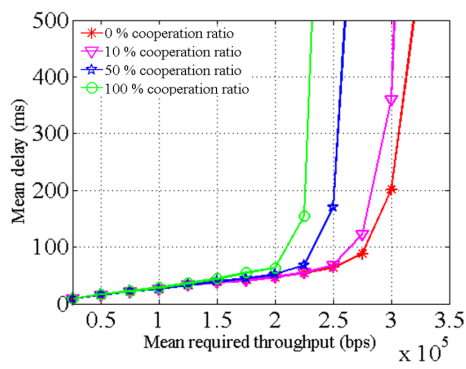

(b) With MaxSNR.

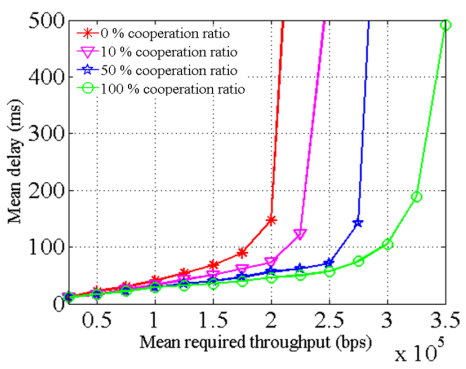

(c) With CEI.

Fig. 1. Measured mobile mean delay with respect to their cooperation ratio.

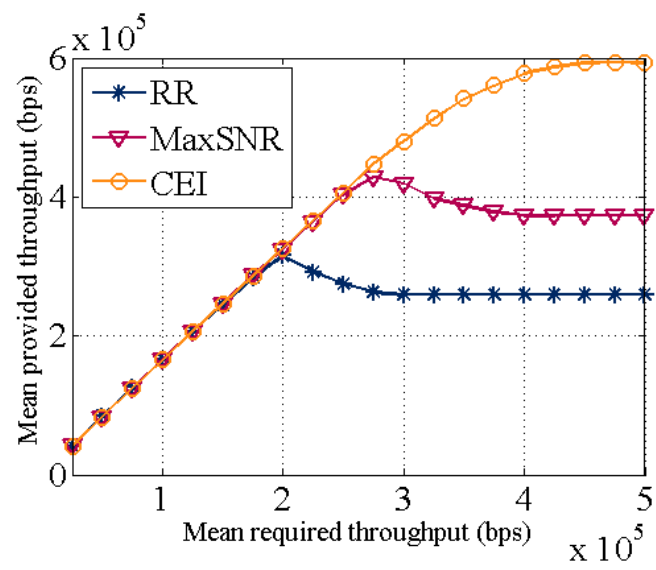

Fig. 2. Relay efficiency.

Figure 1(c) shows the obtained results in the case of the proposed CEI scheduler. We remark that CEI not only encourages the nodes to cooperate but also to enhance the performance in terms of delay. When the nodes increase their cooperation ratio, the enhancement of their delay is more important. For exemple, the nodes with $100 \%$ as cooperative ratio, have a delay inferior to $100 \mathrm{~ms}$ when the mean required throughput is less than $3 \times 10^{5}$ bps which is not possible with other schedulers. The CEI dynamically and gradually adjusts the relative priorities of the mobiles in order to fairly and adequately reward them according to their relative cooperation ratio. With this approach, sparingly delaying the selfish mobiles, the CEI helps the others and whatever the traffic load, the mobile which provides the best cooperative ratio experiences the lowest packet delay.

\section{Relay efficiency impact}

Figure 2 illustrates the relay efficiency in terms of the total mean throughput that each scheduling algorithm has allowed to provide out of the cell. We remark that RR provides the worst performances compared to MaxSNR and CEI. MaxSNR allows to relay more packets but it is the CEI which gives the best number of provided throughputs out of the cell. The RR and MaxSNR curves decrease after the peaks due to the best cooperative mobile penalizing when the system capacity is reached. The CEI, according more priority to friendly mobiles, continues to increase the total amount of forwarding throughputs until a high traffic load which corresponds to a high network extension capacity. With this new resource allocation strategy, when the mean required throughput of each mobile is equal to $500 \mathrm{Kbps}$, the total amount of data transmitted out of the cell in order to extend the coverage area can be increased around 59\% compared to the well acknowledged MaxSNR and around 129\% compared to the classical scheduling algorithm RR.

\section{CONCLUSION}

In this paper we have proposed a new incentive approach which encourages nodes to relay neighboors frame. With our proposition, the mobile stays free to cooperate or not but the proposed scheduler CEI sparingly awards participative nodes so that it is more interesting for them to actively contribute to a good network connectivity. This result is a well-balanced resource allocation which allows to increase the network coverage area while never reducing the global system throughput thanks to a combined opportunistic approach. A minimum throughput is guaranteed to all mobiles of the cell and, thanks to its high spectral efficiency, the mean packet delay provided to the selfish mobiles by CEI is close to the best RR performance. These CEI performance results are all the more interesting that a significant priority is given to mobiles which help the network providing a low packet delay and a high personal throughput. In the future work, we plan to introduce services differentiation in our proposed solution.

\section{REFERENCES}

[1] A. K. Sadek, Z. Han, and K. Ray Liu, "Distributed relay-assignment protocols for coverage expansion in cooperative wireless networks," IEEE Transactions on Mobile Computing, vol. 9, no. 4, pp. 505 - 515, 2010.

[2] A. Florea and H. Yanikomeroglu, "The optimal number of hops in infrastructure-based fixed relay networks," in Proc. IEEE Globecom, St. Louis, MO, Nov. 2005, pp. 3242- 3247.

[3] M. Sikora, J. N. Laneman, M. Haenggi, D. Costello, and T. Fuja, "Bandwidth and power-efficient routing in linear wireless networks," IEEE Transactions Inf. Theory, vol. 52, no. 6, pp. 624- 2633, 2006.

[4] J. Nagle, "On packet switches with infinite storage," IEEE Transactions on Communications, vol. 35, no. 4, pp. 435 - 438, April 1987.

[5] C. Y. Wong and R. S. Cheng, "Multiuser OFDM with adaptive subcarrier, bit, and power allocation," IEEE J. Sel. Areas Commun., 1999.

[6] X. Wang and W. Xiang, "An OFDM-TDMA/SA MAC protocol with QoS constraints for broadband wireless LANs," ACM/Springer Wireless Networks, vol. 12, no. 2, pp. 159 - 170, 2006.

[7] Y. G. Li, N. Seshadri, and S. Ariyavisitakul, "Channel estimation for OFDM systems with transmitter diversity in mobile wireless channels," IEEE J. Sel. Areas Commun., vol. 17, no. 3, pp. 461 - 471, March. 1999.

[8] J. G. Proakis, Digital Communications. 3rd ed. New York: McGraw-Hill, 1995.

[9] J. D. Parsons, The Mobile Radio Propagation Channel. Wiley, 1992. 